Bull. Korean Math. Soc. 52 (2015), No. 2, pp. 649-659

http://dx.doi.org/10.4134/BKMS.2015.52.2.649

\title{
SUBSTITUTION OPERATORS IN THE SPACES OF FUNCTIONS OF BOUNDED VARIATION $B V_{\alpha}^{2}(I)$
}

\author{
Wadie Aziz, José Atilio Guerrero, and Nelson Merentes \\ In memory of Professor José Aguilera

\begin{abstract}
The space $B V_{\alpha}^{2}(I)$ of all the real functions defined on interval $I=[a, b] \subset \mathbb{R}$, which are of bounded second $\alpha$-variation (in the sense De la Vallé Poussin) on $I$ forms a Banach space. In this space we define an operator of substitution $H$ generated by a function $h: I \times \mathbb{R} \longrightarrow \mathbb{R}$, and prove, in particular, that if $H$ maps $B V_{\alpha}^{2}(I)$ into itself and is globally Lipschitz or uniformly continuous, then $h$ is an affine function with respect to the second variable.
\end{abstract}

\section{Introduction}

Let $(F(I), \mathbb{R})$ be the vector space of all the real functions defined on interval $I \subset \mathbb{R}$. Every function $h: I \times \mathbb{R} \rightarrow \mathbb{R}$ generates the Nemytskii (or substitution) operator $H: F(I, \mathbb{R}) \rightarrow F(I, \mathbb{R})$ defined by

$$
(H f)(t)=h(t, f(t)), \quad t \in I .
$$

In many applications to differential, integral, or functional equations, more than just continuity the operator (1.1) is required in order to make use of the basic principles of nonlinear analysis. For instance, in order to solve the functional equation $f(t)=h(t, f(t))$ with respect to $f \in(X,\|\cdot\|) \subset F(I)$, one could try to apply the classical Banach fixed point theorem by imposing on $H: X \longrightarrow X$ a global Lipschitz condition. However, it is well known that this leads to a strong degeneracy for the corresponding function $h$, the global Lipschitz condition on operator (1.1) implies that $h$ must have the form

$$
h(y, y)=f_{0}(t) y+f_{1}(t), \quad t \in I, y \in \mathbb{R},
$$

where $f_{0}, f_{1} \in X$, which means that $h$ must be affine. This phenomenon was proved first by Matkowski $[10,11]$ in the space of Lipschitz continuous or continuously differentiable functions, and by Matkowska, Matkowski and

Received May 1, 2014

2010 Mathematics Subject Classification. Primary 47B33; Secondary 26B30.

Key words and phrases. variation in the sense of De la Vallée Poussin, uniformly continuous operator, Nemytskii (substitution) operator, Jensen equation. 
Merentes [8, 9]. Subsequently, parallel results have been proved in various spaces of functions of bounded variation (cf. [15, 16, 17]).

In this paper, we prove that if $H$ maps the space $B V_{\alpha}^{2}(I)$ of functions of bounded $\alpha$-second variation into itself and it is globally Lipschitz or uniformly continuous, then $h$, the generator function of the operator $H$, is an affine function with respect to the second variable.

This generalizes the results of Kostrzewski [6], where it is assumed that $H$ is globally Lipschitzian. The uniformly continuous composition operators were firstly considered in [12] for the space of differentiable functions and absolutely continuous functions, later in [13] for the space of Hölder function, and in [14] for the space of bounded variation functions. Later, these were used in the main result of the papers $[1,2,3,5]$.

\section{Notation and preliminaries}

In this section we present some necessary notations and definitions and recall some knowledge concerning the bounded $\alpha$-second variation and $\alpha$-derivative.

De la Vallée Poussin (cf. [20]) defined the second variation of a function $f$ on the interval $I=[a, b]$ by

$$
V^{2}(f)=V^{2}(f ; I)=\sup \sum_{j=1}^{n-1}\left|\frac{f\left(t_{j+1}\right)-f\left(t_{j}\right)}{t_{j+1}-t_{j}}-\frac{f\left(t_{j}\right)-f\left(t_{j-1}\right)}{t_{j}-t_{j-1}}\right|,
$$

where the supremum is taken over all partitions $\pi: a=t_{0}<t_{1}<\cdots<t_{n}=b$ of $I$. If $V^{2}(f ; I)<+\infty$, we say that $f$ is of bounded second variation on $I$. The class of all functions which are of bounded second variation is denoted by $B V^{2}(I)$

This class of functions was generalized with respect to a strictly increasing continuous function $\alpha: I \longrightarrow \mathbb{R}$ ( $\alpha$ is called a weight function). Throughout the paper $\alpha$ will be weight function (cf. [19, Definition 3]).

Let $f$ be a real function defined on $I$. For a given partition of the form: $\pi: a=t_{0}<t_{1}<\cdots<t_{n}=b$ of $I$, we set

$$
\sigma_{(2, \alpha)}=\sum_{j=1}^{n-1}\left|f_{\alpha}\left[t_{j}, t_{j+1}\right]-f_{\alpha}\left[t_{j}, t_{j-1}\right]\right|,
$$

where

$$
f_{\alpha}\left[t_{j}, t_{j+1}\right]=\frac{f\left(t_{j+1}\right)-f\left(t_{j}\right)}{\alpha\left(t_{j+1}\right)-\alpha\left(t_{j}\right)}
$$

and

$$
V_{\alpha}^{2}(f)=V_{\alpha}^{2}(f, I)=\sup _{\pi} \sigma_{(2, \alpha)}(f ; \pi),
$$

where the supremum is taken over all partitions $\pi$ of $I$.

If $V_{\alpha}^{2}(f ; I)<\infty$, we say that $f$ is of bounded second $\alpha$-variation on $I$. The set of all these functions will be denoted by $B V_{\alpha}^{2}(I)$. 
A function $f$ is $\alpha$-derivable at $x_{0} \in I$ if $\lim _{x \rightarrow x_{0}} \frac{f(x)-f\left(x_{0}\right)}{\alpha(x)-\alpha\left(x_{0}\right)}$ exists. If this limit exists, we denote its value by $f_{\alpha}^{\prime}\left(x_{0}\right)$, which we call the $\alpha$-derivative of $f$ at $x_{0}$.

Modifying a little the argument (cf. [18, Th. B, p. 24]), we conclude that if $f \in B V_{\alpha}^{2}(I)$, there exist the left $\alpha$-derivative $f_{\alpha^{-}}^{\prime}$ on $(a, b]$ and the right $\alpha$-derivative $f_{\alpha^{+}}^{\prime}$ on $[a, b)$.

The class $B V_{\alpha}^{2}(I)$ is a Banach space equipped with the norm

$$
\|f\|_{\alpha}=|f(a)|+\left|f_{\alpha^{+}}^{\prime}(a)\right|+V_{\alpha}^{2}(f) .
$$

Definition 2.1. A function $f: I \rightarrow \mathbb{R}$ is said to be $\alpha$-Lipschitz if there exists a constant $L>0$ such that

$$
|f(x)-f(y)| \leq L|\alpha(x)-\alpha(y)|, \quad x, y \in I .
$$

By $\alpha$-Lip $(I)$ we will denote the space of functions which are $\alpha$-Lipschitz. If $f \in \alpha$-Lip $(I)$ we define

$$
\operatorname{Lip}_{\alpha}(f)=\sup \left\{\left|\frac{f(x)-f(y)}{\alpha(x)-\alpha(y)}\right|: x \neq y \in I\right\} .
$$

Lemma 2.2. If $f \in B V_{\alpha}^{2}(I)$, then

$$
\operatorname{Lip}_{\alpha}(f) \leq V_{\alpha}^{2}(f)+\left|f_{\alpha^{+}}^{\prime}(a)\right| .
$$

Proof. For all $t_{1}, t_{2}, t_{3} \in I$, with $a<t_{1}<t_{2}<t_{3} \leq b$ we have

$$
\begin{aligned}
& \left|\frac{f\left(t_{3}\right)-f\left(t_{2}\right)}{\alpha\left(t_{3}\right)-\alpha\left(t_{2}\right)}\right|-\left|\frac{f\left(t_{1}\right)-f(a)}{\alpha\left(t_{1}\right)-\alpha(a)}\right| \\
= & \left(\left|\frac{f\left(t_{3}\right)-f\left(t_{2}\right)}{\alpha\left(t_{3}\right)-\alpha\left(t_{2}\right)}\right|-\left|\frac{f\left(t_{2}\right)-f\left(t_{1}\right)}{\alpha\left(t_{2}\right)-\alpha\left(t_{1}\right)}\right|\right)+\left(\left|\frac{f\left(t_{2}\right)-f\left(t_{1}\right)}{\alpha\left(t_{2}\right)-\alpha\left(t_{1}\right)}\right|-\left|\frac{f\left(t_{1}\right)-f(a)}{\alpha\left(t_{1}\right)-\alpha(a)}\right|\right) \\
\leq & \left|\frac{f\left(t_{3}\right)-f\left(t_{2}\right)}{\alpha\left(t_{3}\right)-\alpha\left(t_{2}\right)}-\frac{f\left(t_{2}\right)-f\left(t_{1}\right)}{\alpha\left(t_{2}\right)-\alpha\left(t_{1}\right)}\right|+\left|\frac{f\left(t_{2}\right)-f\left(t_{1}\right)}{\alpha\left(t_{2}\right)-\alpha\left(t_{1}\right)}-\frac{f\left(t_{1}\right)-f(a)}{\alpha\left(t_{1}\right)-\alpha(a)}\right| \\
\leq & V_{\alpha}^{2}(f),
\end{aligned}
$$

so

$$
\left|\frac{f\left(t_{3}\right)-f\left(t_{2}\right)}{\alpha\left(t_{3}\right)-\alpha\left(t_{2}\right)}\right| \leq\left|\frac{f\left(t_{1}\right)-f(a)}{\alpha\left(t_{1}\right)-\alpha(a)}\right|+V_{\alpha}^{2}(f) .
$$

Passing to the limit on the right side in this inequality, as $t_{1} \longrightarrow a^{+}$, we get

$$
\left|\frac{f\left(t_{3}\right)-f\left(t_{2}\right)}{\alpha\left(t_{3}\right)-\alpha\left(t_{2}\right)}\right| \leq\left|f_{\alpha^{+}}^{\prime}(a)\right|+V_{\alpha}^{2}(f) .
$$

and inequality (2.1) follows.

Lemma 2.3 ([4, Th. 4.1]). If $f, g \in B V_{\alpha}^{2}(I)$, then $f \cdot g \in B V_{\alpha}^{2}(I)$. Moreover

$$
V_{\alpha}^{2}(f \cdot g) \leq\|f\|_{\infty} V_{\alpha}^{2}(g)+\|g\|_{\infty} V_{\alpha}^{2}(f)+2(\alpha(b)-\alpha(a)) \operatorname{Lip}_{\alpha}(f) \operatorname{Lip}_{\alpha}(g) .
$$




\section{First main result}

In this section we prove the first main result of the paper, which reads as follows.

Theorem 3.1. If the Nemytskii operator $H$ transforms the space $B V_{\alpha}^{2}(I)$ into itself and $H$ is globally Lipschitzian map, i.e., there is an $L \geq 0$ such that

$$
\left\|H f_{1}-H f_{2}\right\|_{\alpha} \leq L\left\|f_{1}-f_{2}\right\|_{\alpha}, \quad f_{1}, f_{2} \in B V_{\alpha}^{2}(I)
$$

then, there are functions $A, B \in B V_{\alpha}^{2}(I)$ such that

$$
h(t, y)=A(t) y+B(t), \quad t \in I, y \in \mathbb{R} .
$$

Moreover, if $A, B \in B V_{\alpha}^{2}(I)$, then the Nemytskii operator $H$ generated by a function $h$ of the form (3.2) maps $B V_{\alpha}^{2}(I)$ into itself and fulfils inequality (3.1).

Proof. Suppose that the operator $H$ fulfils condition (3.1). Since $H f \in B V_{\alpha}^{2}(I)$ for every function $f \in B V_{\alpha}^{2}(I)$, so putting $f(t)=u$ for $t \in I$, we see that for every $u \in \mathbb{R}$ the function $h(\cdot, u) \in B V_{\alpha}^{2}(I)$. Then $h(\cdot, u)$ is a continuous function. From the definition of the norm and (3.1), we get

$$
\begin{aligned}
& V_{\alpha}^{2}\left(h\left(\cdot, f_{1}(\cdot)\right)-h\left(\cdot, f_{2}(\cdot)\right)\right)+\left|\left(h\left(\cdot, f_{1}(\cdot)\right)-h\left(\cdot, f_{2}(\cdot)\right)\right)_{\alpha^{+}}^{\prime}(a)\right| \\
& +\left|h\left(a, f_{1}(a)\right)-h\left(a, f_{2}(a)\right)\right| \\
\leq & L\left(V_{\alpha}^{2}\left(f_{1}-f_{2}\right)+\left|\left(f_{1}-f_{2}\right)_{\alpha^{+}}^{\prime}(a)\right|+\left|\left(f_{1}-f_{2}\right)(a)\right|\right) .
\end{aligned}
$$

For fixed $t, \bar{t} \in(a, b), t<\bar{t}$ and let $y_{1}, y_{2}, \bar{y}_{1}, \bar{y}_{2} \in \mathbb{R}$ with $y_{1} \neq y_{2} \neq \bar{y}_{1} \neq \bar{y}_{2}$. we define the function $\eta_{t, \bar{t}}: I \rightarrow[0,1]$ by

$$
\eta_{t, \bar{t}}(s)= \begin{cases}y_{i}, & a \leq s<t \\ \frac{\alpha(s)-\alpha(t)}{\alpha(\bar{t})-\alpha(t)}, & t \leq s \leq \bar{t}, \\ \bar{y}_{i}, & \bar{t}<s \leq b .\end{cases}
$$

Consider the functions $f_{i}: I \rightarrow \mathbb{R}$, for $i=1,2$ defined by

$$
f_{i}(\tau):=\eta_{t, \bar{t}}(\tau)\left(\bar{y}_{i}-y_{i}\right)+y_{i} ; \quad \tau \in I, i=1,2
$$

we have that $f_{i} \in B V_{\alpha}^{2}(I)$ for all $i=1,2$; because

$$
V_{\alpha}^{2}\left(f_{i}\right)=2\left|\frac{y_{i}-\bar{y}_{i}}{\alpha(\bar{t})-\alpha(t)}\right| \quad \text { for } i=1,2,
$$

and if $\alpha(s) \nearrow \alpha(\bar{t})$, we obtain

$$
\left\|f_{1}-f_{2}\right\|_{\alpha}=2\left|\frac{y_{1}-y_{2}-\bar{y}_{1}+\bar{y}_{2}}{\alpha(\bar{t})-\alpha(t)}\right|+\left|y_{1}-y_{2}\right| \text {. }
$$

For the functions $f_{1}, f_{2}$ we have the following inequalities:

$$
\begin{aligned}
& V_{\alpha}^{2}\left(h\left(\cdot, f_{1}(\cdot)\right)-h\left(\cdot, f_{2}(\cdot)\right)\right) \\
\geq & \mid \frac{h\left(\bar{t}, f_{1}(\bar{t})\right)-h\left(\bar{t}, f_{2}(\bar{t})\right)-h\left(t, f_{1}(t)\right)+h\left(t, f_{2}(t)\right)}{\alpha(\bar{t})-\alpha(t)}
\end{aligned}
$$




$$
\begin{gathered}
\text { Substitution operator And the SpaCe } B V_{\alpha}^{2}(I) \\
-\frac{h\left(t, f_{1}(t)\right)-h\left(t, f_{2}(t)\right)-h\left(a, f_{1}(a)\right)+h\left(a, f_{2}(a)\right)}{\alpha(t)-\alpha(a)} \mid \\
\geq\left|\frac{h\left(\bar{t}, f_{1}(\bar{t})\right)-h\left(\bar{t}, f_{2}(\bar{t})\right)-h\left(t, f_{1}(t)\right)+h\left(t, f_{2}(t)\right)}{\alpha(\bar{t})-\alpha(t)}\right| \\
-\left|\frac{h\left(t, f_{1}(t)\right)-h\left(t, f_{2}(t)\right)-h\left(a, f_{1}(a)\right)+h\left(a, f_{2}(a)\right)}{\alpha(t)-\alpha(a)}\right| .
\end{gathered}
$$

Taking into consideration (3.4) the above inequality, we see that inequality (3.3) takes the form

$$
\begin{aligned}
& \left|\frac{h\left(\bar{t}, f_{1}(\bar{t})\right)-h\left(\bar{t}, f_{2}(\bar{t})\right)-h\left(t, f_{1}(t)\right)+h\left(t, f_{2}(t)\right)}{\alpha(\bar{t})-\alpha(t)}\right| \\
& -\left|\frac{h\left(t, f_{1}(t)\right)-h\left(t, f_{2}(t)\right)-h\left(a, f_{1}(a)\right)+h\left(a, f_{2}(a)\right)}{\alpha(t)-\alpha(a)}\right| \\
& +\left|\left(h\left(\cdot, f_{1}(\cdot)\right)-h\left(\cdot, f_{2}(\cdot)\right)\right)_{\alpha^{+}}^{\prime}(a)\right|+\left|h\left(a, y_{1}\right)-h\left(a, y_{2}\right)\right| \\
\leq & L\left(2\left|\frac{y_{1}-y_{2}-\bar{y}_{1}+\bar{y}_{2}}{\alpha(t)-\alpha(\bar{t})}\right|+\left|y_{1}-y_{2}\right|\right),
\end{aligned}
$$

equivalently,

$$
\begin{aligned}
& \left|h\left(\bar{t}, f_{1}(\bar{t})\right)-h\left(\bar{t}, f_{2}(\bar{t})\right)-h\left(t, f_{1}(t)\right)+h\left(t, f_{2}(t)\right)\right| \\
& -\left|\frac{h\left(t, f_{1}(t)\right)-h\left(t, f_{2}(t)\right)-h\left(a, f_{1}(a)\right)+h\left(a, f_{2}(a)\right)}{\alpha(t)-\alpha(a)}\right||\alpha(\bar{t})-\alpha(t)| \\
& +\left|\left(h\left(\cdot, f_{1}(\cdot)\right)-h\left(\cdot, f_{2}(\cdot)\right)\right)_{\alpha^{+}}^{\prime}(a)\right||\alpha(\bar{t})-\alpha(t)| \\
& +\left|h\left(a, y_{1}\right)-h\left(a, y_{2}\right)\right||\alpha(\bar{t})-\alpha(t)| \\
\leq & 2 L\left|y_{1}-y_{2}-\bar{y}_{1}+\bar{y}_{2}\right|+L\left|y_{1}-y_{2}\right||\alpha(\bar{t})-\alpha(t)| .
\end{aligned}
$$

Passing to the limits on both sides of this inequality as $\alpha(\bar{t}) \nearrow \alpha(t)$, we get (3.5) $\left|h\left(\bar{t}, f_{1}(\bar{t})\right)-h\left(\bar{t}, f_{2}(\bar{t})\right)-h\left(t, f_{1}(t)\right)+h\left(t, f_{2}(t)\right)\right| \leq 2 L\left|y_{1}-y_{2}-\bar{y}_{1}+\bar{y}_{2}\right|$.

Setting here

$$
y_{1}=w+z, \quad y_{2}=w, \quad \bar{y}_{1}=z, \quad \bar{y}_{2}=0,
$$

we have

$$
|h(\bar{t}, z)-h(\bar{t}, 0)-h(t, w+z)+h(t, w)| \leq 0 .
$$

For any $t \in I$, we define $F_{t}: \mathbb{R} \rightarrow \mathbb{R}$ by the formula

$$
F_{t}(y):=h(t, y)-h(t, 0) .
$$

Hence by (3.6) we have

$$
F_{t}(w+z)=F_{t}(w)+F_{t}(z),
$$


i.e., $F_{t}$ is additive. Setting $\bar{y}_{1}=\bar{y}_{2}=0$ in the (3.5) we have

$$
|h(\bar{t}, z)-h(\bar{t}, 0)-h(t, w+z)+h(t, w)| \leq L\left|y_{1}-y_{2}\right|,
$$

that is

$$
\left|F_{t}\left(y_{2}\right)-F_{t}\left(y_{1}\right)\right| \leq L\left|y_{1}-y_{2}\right|, \quad t \in I, y_{1}, y_{2} \in \mathbb{R},
$$

which implies the continuity of $F_{t}$. Therefore, there exists $A(t) \in \mathbb{R}$ such that

$$
F_{t}(y)=A(t) y, \quad y \in \mathbb{R} .
$$

Setting $B(t)=h(t, 0)$ for $t \in I$, we have by $(3.7)$

$$
h(t, y)=A(t) y+B(t), \quad t \in I, y \in \mathbb{R} .
$$

The function $B \in B V_{\alpha}^{2}(I)$. Since $A(t)=h(t, 1)-h(t, 0)$, so $A \in B V_{\alpha}^{2}(I)$.

Now, suppose that $A, B \in B V_{\alpha}^{2}(I)$ and operator $H$ is generated by the function $h$ defined by (3.2).

It follows from the Lemma 2.3 that $H: B V_{\alpha}^{2}(I) \rightarrow B V_{\alpha}^{2}(I)$. Moreover, for every functions $f_{1}, f_{2} \in B V_{\alpha}^{2}(I)$ and $f:=f_{1}-f_{2}$ we have

$$
\begin{aligned}
\left\|H f_{1}-H f_{2}\right\|_{\alpha}= & \|A f\|_{\alpha}=V_{\alpha}^{2}(A f)+\left|(A f)_{\alpha^{+}}^{\prime}(a)\right|+|(A f)(a)| \\
\leq & \sup _{I}|A| V_{\alpha}^{2}(f)+\sup _{I}|f| V_{\alpha}^{2}(A)+L_{A} L_{f}(\alpha(b)-\alpha(a)) \\
& +\left|A(a) f_{\alpha^{+}}^{\prime}(a)\right|+\left|f(a) A_{\alpha^{+}}^{\prime}(a)\right|+|A(a) f(a)| .
\end{aligned}
$$

By Lemma 2.2 and the inequalities

$|f(t)| \leq|f(t)-f(a)|+|f(a)| \leq L_{f}|\alpha(t)-\alpha(a)|+|f(a)| \leq L_{f}(\alpha(b)-\alpha(a))+|f(a)|$ for all $t \in I$, we obtain

$$
\begin{aligned}
& \left\|H f_{1}-H f_{2}\right\|_{\alpha} \\
\leq & \sup _{I}|A| V_{\alpha}^{2}(f)+|f(a)| V_{\alpha}^{2}(A)+V_{\alpha}^{2}(f) V_{\alpha}^{2}(A)(\alpha(b)-\alpha(a)) \\
& +\left|f_{\alpha^{+}}^{\prime}(a)\right| V_{\alpha}^{2}(A)(\alpha(b)-\alpha(a)) L_{A} V_{\alpha}^{2}(f)(\alpha(b)-\alpha(a)) \\
& +L_{A}\left|f_{\alpha^{+}}^{\prime}(a)\right|(\alpha(b)-\alpha(a))+|A(a)|\left|f_{\alpha^{+}}^{\prime}(a)\right| \\
& +|f(a)|\left|A_{\alpha^{+}}^{\prime}(a)\right|+|A(a)||f(a)| \\
= & {\left[\sup _{I}|A|+V_{\alpha}^{2}(A)(\alpha(b)-\alpha(a))+L_{A}(\alpha(b)-\alpha(a))\right] V_{\alpha}^{2}(f) } \\
& +\left[|A(a)|+V_{\alpha}^{2}(A)(\alpha(b)-\alpha(a))+L_{A}(\alpha(b)-\alpha(a))\right]\left|f_{\alpha^{+}}^{\prime}(a)\right| \\
& +\left[|A(a)|+\left|A_{\alpha^{+}}^{\prime}(a) V_{\alpha}^{2}(A)\right|\right]|f(a)| \\
\leq & {\left[\sup _{I}|A|+V_{\alpha}^{2}(A)(\alpha(b)-\alpha(a))+L_{A}(\alpha(b)-\alpha(a))\right] V_{\alpha}^{2}(f) } \\
& +\left[\sup _{I}|A| V_{\alpha}^{2}(A)(\alpha(b)-\alpha(a))+L_{A}(\alpha(b)-\alpha(a))\right]\left|f_{\alpha^{+}}^{\prime}(a)\right| \\
& +\left[\sup _{I}|A|+V_{\alpha}^{2}(A) L_{A}\right]|f(a)| .
\end{aligned}
$$


Thus setting here

$$
\delta:=\sup _{I}|A| \quad \text { and } \quad \eta:=\max \{1, \alpha(b)-\alpha(a)\},
$$

we get $\left\|H f_{1}-H f_{2}\right\|_{\alpha} \leq L\left\|f_{1}-f_{2}\right\|_{\alpha}$, where $L:=\delta+\eta\left(V_{\alpha}^{2}(A)+L_{A}\right)$.

\section{Uniformly continuous composition operator}

Now, we shall weaken the hypothesis of Theorem 3.1 and we get a proposition that holds only the necessary condition for the Nemytskii operator, for this, we need to recall some definitions and results that we will use for this purpose.

We put

$$
\mathbf{p}(f):=\mathbf{p}(f ; I)=\inf \left\{\epsilon>0: V_{\alpha}^{2}(f / \epsilon) \leq 1\right\}, \quad f \in B V_{\alpha}^{2}(I),
$$

SO

$$
\|f\|_{\alpha}:=|f(a)|+\left|f_{\alpha^{+}}^{\prime}(a)\right|+\mathbf{p}(f) .
$$

Lemma 4.1. Let $f \in B V_{\alpha}^{2}(I)$ and $1<p<+\infty$. We have:

(1) if $\mathbf{p}(f)>0$, then $V_{\alpha}^{2}(f / \mathbf{p}(f)) \leq 1$;

(2) if $\rho>0$, then $V_{\alpha}^{2}(f / \rho) \leq 1$ if and only if $\mathbf{p}(f) \leq \rho$;

(3) if $\rho>0$ and $V_{\alpha}^{2}(f / \rho) \leq 1$, then $\mathbf{p}(f)=\rho$.

Proof. (1) The definition of $\mathbf{p}(f)$ implies $V_{\alpha} \leq 1$ for all $\rho>\mathbf{p}(f)$. Choose a sequence $\rho_{n}>\mathbf{p}(f), n \in \mathbb{N}$, which converges to $\mathbf{p}(f)$ as $n \rightarrow+\infty$. Then $f / \rho_{n} \rightarrow f / \mathbf{p}(f)$ uniformly on $I$. So that

$$
V_{\alpha}^{2}(f / \mathbf{p}(f)) \leq \liminf _{n \rightarrow+\infty} V_{\alpha}^{2}\left(f / \rho_{n}\right) \leq 1
$$

It follows that $\mathbf{p}(f) \in\left\{\rho>0: V_{\alpha}^{2}(f / \mathbf{p}(f)) \leq 1\right\}$ and

$$
\mathbf{p}(f)=\left\{\rho>0: V_{\alpha}^{2}(f / \mathbf{p}(f)) \leq 1\right\}
$$

(2) If $V_{\alpha}^{2}(f / \mathbf{p}(f)) \leq 1$, by definition of $\mathbf{p}(f)$ implies $\mathbf{p}(f) \leq \rho$. If $\mathbf{p}(f)=\rho$, then $V_{\alpha}^{2}(f / \mathbf{p}(f)) \leq 1$ by $(1)$. Let us show that

$$
\text { if } \mathbf{p}(f)<1 \text {, then } V_{\alpha}^{2}(f / \rho)<1 \text {. }
$$

If $\mathbf{p}(f)=0$, then $f$ is a constant function and $V_{\alpha}^{2}(f / \rho)=0$, so, assume that $\mathbf{p}(f)>0$. From the convexity of $V_{\alpha}^{2}(f)$ and the item above we have:

$$
V_{\alpha}^{2}(f / \rho) \leq \mathbf{p}(f) / \rho V_{\alpha}^{2}(f / \mathbf{p}(f)) \leq \mathbf{p}(f) / \rho<1 .
$$

(3) Let $V_{\alpha}^{2}(f / \rho)=1$. By $(2)$, if $\mathbf{p}(f)>\rho$, then $V_{\alpha}^{2}(f)>1$, which is impossible. Taking in account (4.1) we conclude that $\mathbf{p}(f)=\rho$. 
Now, we will prove the second main result of the paper, which reads as follows:

Theorem 4.2. If the Nemytskii operator $H$ transforms the space $B V_{\alpha}^{2}(I)$ into itself and $H$ is uniformly continuous map, i.e.,

$$
\left\|H f_{1}-H f_{2}\right\|_{\alpha} \leq \omega\left(\left\|f_{1}-f_{2}\right\|_{\alpha}\right), \quad f_{1}, f_{2} \in B V_{\alpha}^{2}(I),
$$

where $\omega: \mathbb{R}_{+} \rightarrow \mathbb{R}_{+}$is the modulus continuity of $H$. Then there are functions $A, B \in B V_{\alpha}^{2}(I)$ such that

$$
h(t, y)=A(t) y+B(t), \quad t \in I, y \in \mathbb{R} .
$$

Proof. For every $x \in \mathbb{R}$, the constant function $u(t)=x, t \in I$, belongs to $B V_{\alpha}^{2}(I)$. Since the Nemytskii operator $H$ maps the space $B V_{\alpha}^{2}(I)$ into $B V_{\alpha}^{2}(I)$, it follows that, the function $t \mapsto h(t, u(t))=h(t, x)$ belongs to $B V_{\alpha}^{2}(I)$.

The uniform continuous of $H$ on $B V_{\alpha}^{2}(I)$ implies

$$
\left\|H f_{1}-H f_{2}\right\|_{\alpha} \leq \omega\left(\left\|f_{1}-f_{2}\right\|_{\alpha}\right), \quad f_{1}, f_{2} \in B V_{\alpha}^{2}(I),
$$

where $\omega: \mathbb{R}_{+} \rightarrow \mathbb{R}_{+}$is the modulus continuity of $H$, i.e.,

$$
\omega(\beta):=\sup \left\{\left\|H\left(f_{1}\right)-H\left(f_{2}\right)\right\|_{\alpha}:\left\|f_{1}-f_{2}\right\|_{\alpha} \leq \beta, f_{1}, f_{2} \in B V_{\alpha}^{2}(I)\right\}
$$

for $\beta>0$. From the definitions of the norm $\|\cdot\|_{\alpha}$, we obtain

$$
\mathbf{p}\left(H\left(f_{1}\right)-H\left(f_{2}\right)\right) \leq\left\|H\left(f_{1}\right)-H\left(f_{2}\right)\right\|_{\alpha} \text { for } f_{1}, f_{2} \in B V_{\alpha}^{2}(I) .
$$

Hence, in view of Lemma 4.1 and (4.2), if $\mathbf{p}\left(H\left(f_{1}\right)-H\left(f_{2}\right)\right) \leq \omega\left(\left\|f_{1}-f_{2}\right\|_{\alpha}\right)$, then

$$
V_{\alpha}^{2}\left(\frac{H\left(f_{1}\right)-H\left(f_{2}\right)}{\omega\left(\left\|f_{1}-f_{2}\right\|_{\alpha}\right)}\right) \leq 1 .
$$

Therefore, from definitions of $V_{\alpha}^{2}$ and the operator $H$, for any $f_{1}, f_{2} \in B V_{\alpha}^{2}(I)$ and $\delta, \gamma, \beta \in I, \delta<\gamma<\beta$, we get

$$
\begin{aligned}
& \left|\frac{h\left(\beta, f_{1}(\beta)\right)-h\left(\beta, f_{2}(\beta)\right)-h\left(\gamma, f_{1}(\gamma)\right)+h\left(\gamma, f_{2}(\gamma)\right)}{[\alpha(\beta)-\alpha(\gamma)]}\right| \\
\leq & \omega\left(\left\|f_{1}-f_{2}\right\|_{\alpha}\right)+\left|\frac{h\left(\gamma, f_{1}(\gamma)\right)-h\left(\gamma, f_{2}(\gamma)\right)-h\left(\delta, f_{1}(\delta)\right)+h\left(\delta, f_{2}(\delta)\right)}{[\alpha(\gamma)-\alpha(\delta)]}\right| .
\end{aligned}
$$

For every $y_{1}, y_{2}, \bar{y}_{1}, \bar{y}_{2} \in \mathbb{R}$, the functions $f_{i}: I \longrightarrow \mathbb{R}$ defined by

$$
f_{i}(t)=\frac{y_{i}-\bar{y}_{i}}{\alpha(\gamma)-\alpha(\beta)}(\alpha(t)-\alpha(\gamma))+y_{i}, \quad i \in\{1,2\},
$$

belongs to $B V_{\alpha}^{2}(I)$. Moreover

$$
f_{1}(\beta)=\bar{y}_{1}, \quad f_{2}(\beta)=\bar{y}_{2}, \quad f_{1}(\gamma)=f_{1}(\delta)=y_{1}, \quad f_{2}(\gamma)=f_{2}(\delta)=y_{2}
$$

and

$$
\left\|f_{1}-f_{2}\right\|_{\alpha}=\left|\frac{y_{1}-\bar{y}_{1}-y_{2}+\bar{y}_{2}}{\alpha(\gamma)-\alpha(\beta)}(\alpha(a)-\alpha(\gamma))+y_{1}-y_{2}\right|
$$




$$
+\left|\frac{y_{1}-\bar{y}_{1}-y_{2}+\bar{y}_{2}}{\alpha(\gamma)-\alpha(\beta)} \alpha_{+}^{\prime}(a)\right|
$$

Making the correspond substitutions in (4.3) we obtain

$$
\begin{aligned}
& \left|\frac{\left.h\left(\beta, \bar{y}_{1}\right)-h\left(\beta, \bar{y}_{2}\right)-h\left(\gamma, y_{1}\right)\right)+h\left(\gamma, y_{2}\right)}{[\alpha(\beta)-\alpha(\gamma)]}\right| \\
\leq & \omega\left(\left|\frac{y_{1}-\bar{y}_{1}-y_{2}+\bar{y}_{2}}{\alpha(\gamma)-\alpha(\beta)}(\alpha(a)-\alpha(\gamma))+y_{1}-y_{2}\right|+\left|\frac{y_{1}-\bar{y}_{1}-y_{2}+\bar{y}_{2}}{\alpha(\gamma)-\alpha(\beta)} \alpha_{+}^{\prime}(a)\right|\right) \\
& +\left|\frac{h\left(\gamma, y_{1}\right)-h\left(\gamma, y_{2}\right)-h\left(\delta, y_{1}\right)+h\left(\delta, y_{2}\right)}{[\alpha(\gamma)-\alpha(\delta)]}\right| .
\end{aligned}
$$

Taking arbitrary $u, v \in \mathbb{R}$ and setting in this inequality

$$
y_{1}=\bar{y}_{2}=\frac{u+v}{2}, \quad \bar{y}_{1}=u, \quad y_{2}=v
$$

result

$$
\begin{aligned}
& \left.\mid h(\beta, u)-h\left(\beta, \frac{u+v}{2}\right)-h\left(\gamma, \frac{u+v}{2}\right)\right)+h(\gamma, v) \mid \\
\leq & \omega\left(\mid \frac{u-v}{2}\right)|\alpha(\beta)-\alpha(\gamma)| \\
& +\left|h\left(\gamma, \frac{u+v}{2}\right)-h(\gamma, v)-h\left(\delta, \frac{u+v}{2}\right)+h(\delta, v)\right|\left|\frac{\alpha(\beta)-\alpha(\gamma)}{\alpha(\gamma)-\alpha(\delta)}\right| .
\end{aligned}
$$

Letting $\beta$ tend to $\gamma$ and making use of the continuity of $h$, we hence get

$$
h\left(\gamma, \frac{u+v}{2}\right)=\frac{h(\gamma, u)+h(\gamma, v)}{2}
$$

for all $\gamma \in I$. This shows that for any $t \in I$, the function $h(t, \cdot)$ is Jensen and, by assumptions, it is continuous. Consequently (cf. [7, Th. 1, p. 315]), there exists functions $A, B: I \longrightarrow \mathbb{R}$ such that

$$
h(t, y)=A(t) y+B(t), \quad t \in I, y \in \mathbb{R} .
$$

Since $h(\cdot, y) \in B V_{\alpha}^{2}(I)$ for all $y \in \mathbb{R}$, and, by (4.4),

$$
B(t)=h(t, 0), \quad A(t)=h(t, 1)-B(t), \quad t \in I,
$$

therefore $A, B \in B V_{\alpha}^{2}(I)$.

Acknowledgements. Wadie Aziz was a visiting professor, during the 2013, at the Mathematics School, and his research was inscribed at the CIMPA by Vicerrectoría de Investigación of Universidad de Costa Rica, under the project 821-B3-182.

J. A. Guerrero partially funded by the Decanato de Investigación-Universidad Nacional Experimental del Táchira-Venezuela.

The authors express his gratitude to the referee for his valuable comments. 


\section{References}

[1] A. Acosta, W. Aziz, J. Matkowski, and N. Merentes, Uniformly continuous composition operator in the space of $\varphi$-variation functions in the sense of Riesz, Fasc. Math. 43 (2010), 5-11.

[2] W. Aziz, A. Azocar, J. Guerrero, and N. Merentes, Uniformly continuous composition operator in the space of functions of $\varphi$-variation with weight in the sense of Riesz, Nonlinear Anal. 74 (2011), no. 2, 573-576.

[3] W. Aziz, J. Guerrero, and N. Merentes, Uniformly continuous set-valued composition operators in the spaces of functions of bounded variation in the sense of Riesz, Bull. Pol. Acad. Sci. Math. 58 (2010), no. 1, 39-45.

[4] R. Castillo and E. Trousselot, An application of the generalized Maligranda-Orlicz's Lemma, J. Inequal. Pure Appl. Math. 9 (2008), no. 3, Article 84, 6 pp.

[5] J. A. Guerrero, H. Leiva, J. Matkowski, and N. Merentes, Uniformly continuous composition operators in the space of bounded $\varphi$-variation functions, Nonlinear Anal. $\mathbf{7 2}$ (2010), no. 6, 3119-3123.

[6] T. Kostrzewski, Globally Lipschitz operators of substitution in Banach space BCI, Sci. Bull. Łódzka Technical Univ. 602, (1993), 17-25.

[7] M. Kuczma, An Introduction to the Theory of Functional Equations and Inequalities, Polish Scientific Editors and Silesian University, Warszawa-Kraków-Katowice, 1985.

[8] A. Matkowska, On characterization of Lipschitizian operators of substitution in the class of Hölder functions, Sci. Bull. Łódzka Technical Univ. 17 (1984), 81-85.

[9] A. Matkowska, J. Matkowski, and N. Merentes, Remark on globally Lipschitizian composition operators, Demonstratio Math. 28 (1995), no. 1, 171-175.

[10] J. Matkowski, Functional equations and Nemytskii operators, Funkcial. Ekvac. 25 (1982), no. 2, 127-132.

[11] Form of Lipschitz operators of substitution in Banach spaces of differentiable functions, Sci. Bull. Łódzka Tech. Univ. 17, (1984), 5-10.

[12] _ Uniformly continuous superposition operators in space of differentiable function and absolutely continuous functions, Internat. Ser. Numer. Math. 157 (2008), 155-166.

[13] Uniformly continuous superposition operators in Banach space of Hölder functions, J. Math. Anal. Appl. 359 (2009), no. 1, 56-61.

[14] Uniformly continuous superposition operators in the space of bounded variation functions, Math. Nachr. 283 (2010), no. 7, 1060-1064.

[15] J. Matkowski and J. Miś, On a characterization of Lipschitzian operators of substitution in the space $B V[a, b]$, Math. Nachr. 117 (1984), 155-159.

[16] N. Merentes, On a characterization of Lipschitzian operators of substitution in the space of bounded Riesz $\varphi$-variation, Ann. Univ. Sci. Budapest. Eötvös Sect. Math. 34 (1991), 139-144.

[17] N. Merentes and S. Rivas, On a characterization of the Lipschitzian composition operators between spaces of functions of bounded p-variation, Czechoslov. Math. J. 45 (1995), no. $4,43-48$.

[18] A. W. Roberts and D. E. Varberg, Convex Functions, New York and London, 1973.

[19] A. M. Russell, Functions of bounded second variation and Stieltjes-type integrals, J. Lond. Math. Soc. (2) 2 (1970), 193-208.

[20] Ch. J. de la Vallée Poussin, Sur la convergence des formules d'interpolation entre ordonées équidistantes, Acad. Roy. Belge. Bull. Cl. Sci. (6) 4 (1908), 319-410.

WADIE AZIZ

UNIVERSIDAD DE LOS ANDES

Departamento de Física y Matemática

TRuJILlO-VENEZUELA

E-mail address: wadie@ula.ve 
José Atilio Guerrero

Universidad Nacional Experimental del Táchira

Departamento de Matemática y Física

San Cristóbal-Venezuela

E-mail address: jaguerrero4@gmail.com

Nelson Merentes

Universidad Central de Venezuela

Escuela de MATEMÁticas

CaracAs-Venezuela

E-mail address: nmer@ciens.ucv.ve 